\title{
Prevalencia de desnutrición en niños tarahumaras menor es de cinco años en el municipio de Guachochi, Chihuahua
}

Joel Monárrez, M.C., M. en C.,1, ${ }^{(1,2)}$ Homero Martínez, M.C., Ph.D. ${ }^{(2,3)}$

\author{
Monárrez J, Martínez $\mathrm{H}$. \\ Prevalencia de desnutrición en niños \\ tarahumaras menores de cinco años \\ en el municipio de Guachochi, Chihuahua. \\ Salud Publica Mex 2000;42:8-16.
}

\section{Resumen}

Objetivo. Determinar la prevalencia de desnutrición en niños tarahumaras menores de cinco años en el municipio de Guachochi, C hihuahua, México. Material y métodos Durante el verano de 1996, fueron muestreados 450 niños, representativos de 62 comunidades, estratificadas por el tamaño de su población. La muestra representa $12.2 \%$ del total de tarahumaras del municipio. Para cada uno de los índices antropométricos peso/edad, peso/talla y talla/edad se calculó la desviación en puntaje $Z$ de cada observación de la mediana de la población de referencia (N CHS). Se presentan medias y desviaciones estándar de las mediciones realizadas, así como la prevalencia de desnutrición, estratificada por edad, sexo y tamaño de la localidad de residencia de los niños. Resultados $L a s$ prevalencias de desnutrición $a<-2 Z$ fueron: peso/edad: $36.4 \%$ ( $\pm \pm D E:-1.66 \pm 1.1$ ); peso/talla: 3.5\% ( $\mathrm{X} \pm \mathrm{DE}$ : $-0.43 \pm 0.9)$ y; talla/edad: $57.1 \%(\bar{X} \pm D E:-2.15 \pm 1.3)$. Los niños de $12-23$ meses de edad fueron los más afectados $(<-2 Z)$ de acuerdo con los indicadores peso/talla y peso/edad con $10.3 \%$ y $52.6 \%$, respectivamente. Los grupos de edad mayo res de un año mostraron prevalencias superiores a $60 \%$ en el indicador talla-edad (<-2Z). Los niños mostraron mayor afectación que las niñas $(p<0.05)$. Conclusiones El grupo de edad con mayor afectación por la desnutrición corresponde a los niños de 12 a 23 meses mostrando mayor incidencia en los hombres que en las mujeres. Esta información podrá ser útil para la planeación e instrumentación de intervenciones nutricionales dirigidas a este grupo indígena.

Palabras clave: desnutrición proteico-energética/prevalencia; infante; grupos étnicos; México

\author{
Monárrez J, Martínez $\mathrm{H}$. \\ Prevalence of malnutrition \\ in Tarahumara children under 5 years \\ of age in the municipality of Guachochi, Chihuahua. \\ Salud Publica Mex 2000;42:8-16.
}

\begin{abstract}
A bstract
Objective. To determine the prevalence of malnutrition among Tarahumara children under 5 years of age in the municipality of G uachochi,C hihuahua, Mexico. Material and methods. During the summer of 1996, we interviewed a representative sample of 450 children from 62 communities, stratified by size of population. The sample represents $12.2 \%$ of the total number of Tarahumaras in the municipality. For each of three anthropometric indices (weight-forage, weight-for-height and height-for-age), we calculated the Z-score in reference to N CHS population values. Results are presented as means and standard deviations as well as prevalence of malnutrition at different cut-off-points, stratified by age, sex, and size of the community. Results. Prevalence figures of malnutrition ( $<-2 Z)$ were: weightfor-age: $36.4 \%$ (mean SD $\pm-1.66 \pm 1.1$ ); weight-for-height: $3.5 \%$ (mean SD $\pm-0.43 \pm 0.9$ ); and height-for-age: $57.1 \%$ (mean $\mathrm{SD} \pm-2.15 \pm 1.3)$. C hildren aged $12-23$ months were the most affected, both in weight-for-height and weight-for-age $(10.3 \%$ and $52.6 \%$, respectively). C hildren over one year of age presented prevalences of height-for-age $<-2 Z$ over $60 \%$. Significant sex differences $(p<0.05)$ were observed, favoring girls. Conclusions. Malnutrition among Tarahumara children reaches its peak during the second year of life (12-23 months old), affecting boys more than girls. This information may be useful for planning and targeting nutrition inter vention programs for this underprivileged indigenous group.
\end{abstract}

Key words: protein-energy malnutrition/prevalence; child, preschool; ethnic groups; Mexico

Estudio financiado por el Fondo Social del Empresariado Chihuahuense y el Instituto Tecnológico y de Estudios Superiores de Monterrey, Campus Chihuahua, México.

(1) Coordinación de Investigación, Instituto Tecnológico y de Estudios Superiores de Monterrey, Campus Chihuahua, Chihuahua, México.

(2) Instituto $N$ acional de Salud Pública, México.

(3) División de Investigación Epidemiológica y en Servicios de Salud, Instituto Mexicano del Seguro Social, México.

Fecha de recibido: 13 de julio de 1999 - Fecha de aprobado: 9 de noviembre de 1999 Solicitud de sobretiros: Dr. Joel Monárrez Espino. Burggrafenstr 92, 45139 Essen, Alemania. Correo electrónico: monarrezj@ aol.com 
os tarahumaras, conocidos en su idioma como rarámuri, denotan al grupo étnico que habita el suroeste del estado norteño de Chihuahua, México. Constituye, a su vez, el grupo indígena más numeroso del norte del país, con aproximadamente 80000 habitantes, que representa cerca de $3 \%$ de la población estatal y $1.1 \%$ del total nacional de grupos étnicos indígenas; asimismo, ocupa $88 \%$ del total de indígenas en el estado. ${ }^{1,2}$

Los rarámuri habitan una extensa llanura elevada dividida por barrancos y cañones. El clima es contrastante, de acuerdo con la altitud de cada zona: en la montaña (>2 $000 \mathrm{msnm}$ ) es templado-semifrío, mientras que en el barranco ( $<1000 \mathrm{msnm}$ ) es sub-tropical; las temperaturas medias anuales son de 8 y $24^{\circ} \mathrm{C}$, respectivamente. ${ }^{3}$ Asimismo, la variabilidad de las precipitaciones es notable, al igual que la presencia de sequías frecuentes. Cuando no hay sequía en la zona montañosa, las precipitaciones promedian 600-800 mm. La gran mayoría de las lluvias suceden entre junio y agosto lo que constituye la base de una agricultura de temporal. ${ }^{4}$

Aunque las condiciones agrícolas de la Sierra Tarahumara no son muy adecuadas, producto de los delgados suelos con pobre capacidad para absorber humedad y fertilizantes, ${ }^{5}$ los rarámuri practican la agricultura de subsistencia; cultivan maíz, frijol, papa, chícharo, haba y calabaza. El rendimiento de cultivo de maíz es de $300-450 \mathrm{~kg}$ por hectárea en años con precipitaciones elevadas para la zona, pero apenas suficientes para la subsistencia. Las cosechas se dan en pequeñas zonas de suelo cultivable, que pueden estar separadas unas de otras por varios kilómetros. Dado que el maíz rara vez dura todo el año, las familias se ayudan con la recolección de plantas silvestres como el maquásori (quelite), la cría de pollos y chivos, que son prácticas comunes de subsistencia en esta región. Los recursos económicos requeridos para comprar productos externos a estas comunidades provienen de la venta de animales o artesanías, o del eventual salario que algunos indígenas perciben al emplearse como peones en aserraderos. Por siglos los tarahumaras han sido explotados, primero en las minas y campos de españoles, durante la época colonial, y después por mestizos y extranjeros. ${ }^{4,6}$

Los asentamientos tarahumaras son rancherías que se conforman por grupos dispersos de viviendas. Cada vivienda consiste en un cuarto edificado de troncos o piedras y una o dos chozas para almacenar el maíz. La vivienda es un cuarto/habitación con piso de tierra y una rudimentaria chimenea que hace las veces de cocina; la disponibilidad de agua entubada, elec- tricidad y drenaje es prácticamente inexistente. ${ }^{7}$ La movilidad estacional es común; durante los meses cálidos, las familias permanecen en las tierras altas de la sierra, en tanto que en los inviernos extremadamente fríos, emigran a las partes más bajas. Los tarahumaras de las regiones montañosas viven en rancherías y utilizan los poblados grandes como centros políticos y religiosos. Estas poblaciones, habitadas en su mayoría por mestizos, generalmente cuentan con una iglesia, una escuela, un centro de salud, algún recinto utilizado como corte de justicia o sitio de reunión y pequeñas tiendas operadas por mestizos.

En lo que respecta a la salud, se debe considerar que la recolección de información en una población de difícil acceso, con alta movilidad y barreras de idioma, sumado a los pobres intentos de las autoridades por obtener dicha información, produce estadísticas poco precisas y no actualizadas con un alto grado de subregistro. Sin embargo, aun con estas limitaciones, los indicadores existentes para la población tarahumara muestran diferencias enormes con relación a los estatales. ${ }^{1}$ Los servicios de salud ofrecidos por diversos organismos como el Instituto Mexicano del Seguro Social-Solidaridad, Secretaría de Salud, Instituto Nacional Indigenista, Sistema Nacional para el Desarrollo Integral de la Familia (IMSS-Solidaridad, SSA, INI, DIF) y diversas clínicas religiosas tienen un alcance limitado, se ubican generalmente en los poblados más grandes, son pocos en número y recursos, y se dedican principalmente a la medicina curativa. Mientras que en 1990 la tasa de mortalidad infantil general en el ámbito nacional fue de 35 por 1000 , y la tasa de mortalidad indígena también nacional fue de 55 por 1000 , la mortalidad infantil tarahumara ajustada fue de 95.3 por 1000 nacidos vivos, lo que la ubica como la más alta (junto a huicholes) entre los grupos étnicos indígenas del país. ${ }^{8,9}$

En años recientes, y en particular a partir del otoño de 1994, aparecieron publicados en periódicos estatales varios informes hospitalarios que advertían sobre un incremento en el número de muertes de niños tarahumaras con desnutrición moderada o grave, producto del conocido efecto potenciador de la desnutrición sobre la mortalidad infantil. ${ }^{10-12}$ Las autoridades de salud crearon un programa de intervención nutricional basado en la ayuda alimentaria directa, a través de una canasta de alimentos, así como en la oferta de atención médica en forma ambulatoria. Sin embargo, la planeación y ejecución de dicho programa contó con las limitaciones de la falta de información en el ámbito poblacional que diera una idea precisa de la magnitud del problema nutricio entre los niños rará- 
muri, y que permitiera una distribución eficiente y dirigida de los recursos, así como la subsecuente evaluación del impacto del programa.

El objetivo del presente estudio fue realizar un diagnóstico del estado nutricional de la población infantil tarahumara menor de cinco años con base en la toma de mediciones antropométricas y examen físico.

\section{Material y métodos}

El estudio se llevó a cabo en el municipio de Guachochi, Chihuahua, que cuenta con un tercio $(\approx 3700)$ del total de tarahumaras menores de cinco años en el estado. ${ }^{1}$ La Secretaría de Salud ubica a Guachochi en el primer lugar de mortalidad infantil entre todos los municipios del país, probablemente como efecto del $61.3 \%$ de población rarámuri que lo habita.

El trabajo de campo se realizó entre el 20 de junio y el 30 de agosto de 1997. Este periodo de verano, que corresponde a la época de precosecha, fue elegido tanto para evitar el problema de migración estacional de las familias como para eliminar la variación estacional en el estado antropométrico de los niños dado por la época de pre y poscosecha. ${ }^{13-15}$ La población de estudio consistió en los niños menores de cinco años de ambos sexos, residentes permanentes del municipio, cuyo padre o madre hablase rarámuri.

El tamaño de la muestra fue determinado al considerar la frecuencia estimada de la desnutrición, el nivel de precisión deseado y la factibilidad económica. Se aplicó una fórmula para calcular la proporción de una variable en una población. ${ }^{16}$ Se buscó maximizar el tamaño de la muestra incorporando un factor de 0.5 como indicador de máxima heterogeneidad del fenómeno de estudio, con un nivel de confianza de $95 \%$ y un error relativo del estimador de $15 \%$. Posteriormente, se sumó 30\% (determinado por la factibilidad económica) por el efecto de diseño dado que la muestra no fue obtenida a partir de un muestreo simple aleatorio. El tamaño muestral final fue de 450 niños.

Aunque hubiera sido deseable realizar un muestreo probabilístico de la población, no fue posible contar con un marco muestral debido a que no existe un censo específico para población tarahumara, y la factibilidad de realizarlo se enfrentó con la imposibilidad de ubicar físicamente a la población. Por un lado, la posibilidad de llegar a las localidades aisladas con menos de 10 viviendas (cerca de 1000 en el municipio) era baja, producto de la pobre información cartográfica y de las dificultades logísticas existentes. Por otra parte, dado que las localidades con más de 50 viviendas no cuentan con mapas que distingan las viviendas indígenas de las mestizas, la dificultad logística para identificar dichas viviendas resultaría un esfuerzo censal fuera de los alcances de los investigadores, en las localidades con más de 100 viviendas. Ante estas dificultades, se trazó la siguiente estrategia para buscar la representatividad de la población.

Se asumió que el tamaño de la localidad en la zona tarahumara, dado por el número de viviendas habitadas, guarda relación con la marginalidad de la población. Bajo este supuesto empírico, se definieron tres estratos de acuerdo con el número de viviendas por localidad. La cantidad de niños a ser muestreados se determinó de acuerdo con una estimación de la proporción de tarahumaras que viven en los diferentes estratos.

Las rancherías con menos de 10 viviendas se encuentran distribuidas en la periferia de las rancherías medianas, variando desde 1-2 hasta ocho o más. La distancia de las rancherías chicas a las medianas es de $1 \mathrm{~km}$ a 10 o más, que caminando representan de 30 minutos hasta cinco o más horas. El acceso es por medio de veredas o senderos de montaña, a manera de rutas, rara vez transitables con vehículo. Cada ruta cubre varias rancherías chicas, aunque no necesariamente todas las circundantes a la ranchería mediana. Se eligió aleatoriamente una ruta que partiera de la ranchería mediana seleccionada y siguiendo la ruta elegida, se seleccionaron todas las viviendas con niños menores de cinco años hasta cubrir la cuota. De esta forma se muestrearon 38 rancherías chicas.

Para seleccionar las rancherías medianas se siguió un muestreo sistemático con base en el siguiente esquema: se realizó un listado con cuatro columnas; en la primera, se colocaron en orden alfabético las 114 rancherías medianas del municipio; en la segunda, se listó el número de viviendas por localidad; en la tercera, la frecuencia acumulada de viviendas; y en la cuarta, el rango de viviendas desde la localidad anterior hasta la presente. Se determinó el intervalo muestral del total de viviendas a ser muestreadas a partir del tamaño muestral (por ser la vivienda la unidad muestral). La selección de las localidades se efectuó tomando un número aleatorio al que se sumó el intervalo muestral consecutivamente. Las localidades seleccionadas fueron aquellas en las que el intervalo muestral cayó dentro del rango de viviendas. Al interior de la localidad se eligieron ocho viviendas al azar y se encuestó a todos los niños menores de cinco años que se encontraron. Siguiendo este procedimiento, se eligieron 17 rancherías medianas.

A partir del listado de poblaciones grandes en el municipio, se seleccionó en forma aleatoria a la mitad de ellas. Una vez en la localidad, se recurrió a las promotoras de salud locales para hacer un llamado a to- 
das las madres de niños rarámuri menores de cinco años, solicitando que acudieran al centro de salud, dispensario o escuela en una fecha predeterminada. De las que acudieron, se eligieron al azar los niños necesarios para cubrir la cuota del estrato. Fueron muestreadas siete poblaciones grandes.

La toma de mediciones antropométricas fue llevada a cabo por personal médico según las técnicas descritas y recomendadas por la Organización Mundial de la Salud (OMS). ${ }^{17}$ Los encuestadores que recogieron la información antropométrica fueron estandarizados por un supervisor externo, siguiendo el método propuesto por Habicht. ${ }^{18}$ Como patrón de referencia se utilizaron las tablas del Centro Nacional de Estadísticas de la Salud de Estados Unidos de América, recomendadas por la OMS, para realizar comparaciones internacionales. ${ }^{19}$

Para la obtención del peso se emplearon básculas de resorte tipo Salter con capacidad de $20 \mathrm{~kg}$ y precisión de $100 \mathrm{~g}$, calibradas cada tres mediciones. Para la obtención de la talla se usaron infantómetros de madera y aluminio con precisión de $1 \mathrm{~mm}$.

Como los índices antropométricos dependientes de la edad se ven limitados cuando la edad no se conoce de manera precisa, ${ }^{20}$ se prestó particular importancia a su cálculo. La edad se obtuvo en $61.3 \%$ de los niños a partir de la cartilla de vacunación o el acta de nacimiento; en $35.8 \%$ fue informada por la madre o el padre, y sólo en $2.9 \%$ se estimó mediante la relación de eventos relevantes en la comunidad. En las edades referidas por los padres no se identificó preferencia de dígitos, y fueron similares a las de los documentos oficiales. Esto hace suponer que la edad fue calculada con precisión.

Para identificar las localidades y viviendas se utilizaron los mapas más detallados disponibles de la zona y un localizador geográfico satelital para asegurar la ubicación de las localidades seleccionadas, así como para encontrar caminos y brechas de acceso. Para el trabajo de campo se contrataron traductores-guías locales.

Además de emplear el criterio antropométrico se realizó un examen físico para identificar aquellos niños con desnutrición funcional. ${ }^{21}$ Se evaluaron los siguientes signos presuntivos de desnutrición caloricoproteínica:22 atrofia muscular, edema y alteraciones del cabello (despigmentación, alisamiento, fácilmente desprendible). Se clasificó como marasmáticos a aquellos niños que presentaron el cuadro clínico descrito por Golden. ${ }^{23}$ Además, para valorar la deficiencia de iodo y vitamina A, se evaluó la presencia de bocio aparente y de xerosis conjuntival. Un solo médico realizó el examen físico de todos los niños.
El procesamiento de la información incluyó la revisión de encuestas para identificar inconsistencias o faltantes en la información contenida. La información se capturó en una base de datos empleando DBASE Win ${ }^{\circledR}$, misma que fue cotejada contra las encuestas originales. Se calcularon los índices antropométricos peso/edad, peso/talla y talla/edad mediante el programa ANTHRO 1.01, recurriendo a la desviación de cada observación de la mediana de la población de referencia (puntuación Z). Para el análisis estadístico de la información se empleó SPSS Win ${ }^{\circledR}$. Se calcularon medias y desviaciones estándar así como la prevalencia de desnutrición usando puntos de corte para los tres indicadores antropométricos. La información fue estratificada por grupos de edad, sexo y número de viviendas en la localidad.

\section{Resultados}

Se entrevistaron 450 niños, que representan $12.2 \%$ del total de tarahumaras menores de cinco años del municipio y $4.1 \%$ del total estatal.

La OMS ha recomendado el uso de la puntuación Z como la forma adecuada para analizar la información de antropometría, en función del manejo estadístico que permite. ${ }^{24} \mathrm{El}$ cuadro I muestra la media de la puntuación $Z$ de los tres índices antropométricos expresados, por sexo. Con excepción del índice talla/ edad que presentó un valor de $p=0.08$, los demás índices mostraron que los hombres tuvieron puntuaciones $\mathrm{Z}$ significativamente más bajas que las de las mujeres $(p<0.05)$.

En el cuadro II se muestra la media del puntaje $Z$ de los tres índices antropométricos, de acuerdo con el tamaño de la localidad en la que se encuestó a los niños. Los niños que habitan en las rancherías grandes mostraron los puntajes $Z$ más altos en comparación con los niños de rancherías medianas y pequeñas, aunque esta diferencia no alcanzó significancia estadística en

\section{Cuadro I \\ Media del PUNTAJe $Z$ de LOS INDICAdores ANTROPOMÉTRICOS DE TARAHUMARAS MENORES DE CINCO AÑOS, GLOBAL Y POR SEXO. Guachochi, Chinuahua, México, 1997}

\begin{tabular}{lcccc} 
Indicador & $\begin{array}{c}\text { Global } \\
(\mathrm{n}=450)\end{array}$ & $\begin{array}{c}\text { Hombres } \\
(\mathrm{n}=228)\end{array}$ & $\begin{array}{c}\text { Mujeres } \\
(\mathrm{n}=222)\end{array}$ & $p$ \\
Peso/edad & -1.66 & -1.79 & -1.51 & .00 \\
\hline Peso/talla & -0.43 & -0.52 & -0.33 & .02 \\
\hline Talla/edad & -2.15 & -2.26 & -2.04 & .08
\end{tabular}


el caso del peso para la talla. El indicador que mostró la mayor afectación fue la talla para la edad, seguido del peso para la edad.

El cuadro III presenta porcentajes de la puntuación $Z$ de los tres indicadores antropométricos usando puntos de corte. Se observó que $0.4 \%$ de los niños encuestados mostró un peso para la talla por debajo de $-3 Z$, en tanto que $3.1 \%$ se encontró entre -2 y $-3 Z$. En relación a la talla para edad, $25.1 \%$ estuvo por debajo de $-3 Z$, y $32 \%$ entre -2 y $-3 Z$. En cuanto al peso para la edad, $10.2 \%$ se encontró por debajo de $-3 Z$, y $26.2 \%$ entre -2 y $-3 Z$.

El cuadro IV muestra la frecuencia de los signos clínicos de desnutrición en los niños. De 2.2\% de los niños diagnosticados como marasmáticos, $90 \%$ presentó un peso para la edad $<-2 \mathrm{Z}$ y de $8.8 \%$ que presentó pérdida muscular, $75 \%$ mostró un peso para la edad de <-2Z. Mostraron xerosis conjuntival 2.4\% y ninguno mostró bocio aparente.

\section{Cuadro II}

Media del PUNTAJE Z DE LOS INDICADORES ANTROPOMÉTRICOS* DE TARAHUMARAS MENORES DE CINCO AÑOS, POR TAMAÑO DE LA LOCALIDAD. ${ }^{\ddagger}$ Guachochi, Chihuahua, México, 1997

\begin{tabular}{|c|c|c|c|}
\hline Indicador & $\begin{array}{c}\text { Rancherías } \\
\text { pequeñas } \\
(n=135)\end{array}$ & $\begin{array}{c}\text { Rancherías } \\
\text { medianas } \\
(n=174)\end{array}$ & $\begin{array}{c}\text { Rancherías } \\
\text { grandes } \\
(n=141)\end{array}$ \\
\hline Peso/edad & $-1.73^{a}$ & $-1.76^{a}$ & $-1.46^{b}$ \\
\hline$\overline{P e s o / t a l l a ~}$ & $-0.35^{a}$ & $-0.54^{a}$ & $-0.34^{\mathrm{a}}$ \\
\hline Talla/edad & $-2.31^{\mathrm{a}}$ & $-2.21^{\mathrm{a}}$ & $-1.90^{b}$ \\
\hline \multicolumn{4}{|c|}{$\begin{array}{l}\text { * Los puntajes con supraíndices diferentes (a y b) muestran diferenci } \\
\text { estadísticamente significativa, } p<0.05 \text {, entre sí, comparando por indica } \\
\text { dor entre rancherías (evaluada mediante la prueba de Bonferroni) } \\
\text { ₹ El estrato de ranchería está dado por el número de viviendas habitadas } \\
\text { rancherías pequeñas }<10 \text { viviendas; rancherías medianas } 10-50 \text { vivien } \\
\text { das; rancherías grandes }>50 \text { viviendas }\end{array}$} \\
\hline
\end{tabular}

Cuadro III

Porcentajes de tarahumaras menores DE CINCO AÑOS REFERIDOS A PUNTUACIÓN Z PARA LOS INDICADORES ANTROPOMÉTRICOS. Guachochi, Chinuahua, México, 1997

\begin{tabular}{lrrrr} 
Indicador & $<-3 Z$ & -2 a $-3 Z$ & -2 a $2 Z$ & $>2 Z$ \\
Peso/edad & 10.2 & 26.2 & 63.3 & 0.3 \\
\hline Talla/edad & 25.1 & 32.0 & 42.2 & 0.7 \\
\hline Peso/talla & 0.4 & 3.1 & 95.8 & 0.7
\end{tabular}

La figura 1 muestra porcentajes de niños a $<-2 Z$ para los tres indicadores antropométricos por grupos de edad. Los niños de 12-23 meses de edad fueron los más afectados de acuerdo con los indicadores peso/talla y peso/edad con $10.3 \%$ y $52.6 \%$, seguidos de los niños de $6-11$ meses con $6.1 \%$ y $40.8 \%$, respectivamente. El indicador talla/edad mostró una tendencia creciente hasta el segundo año de edad, para después estabilizarse. Después de un año todos los grupos de edad presentaron prevalencias cercanas a $65 \%$ de déficit de talla a $<-2 Z$.

\section{Cuadro IV \\ Prevalencia de signos positivos DE DESNUTRICIÓN POR EVALUACIÓN CLÍNICA DE TARAHUMARAS MENORES DE CINCO AÑOS. Guachochi, Chinuahua, México, 1997}

\begin{tabular}{lcc} 
Signo clínico & $\begin{array}{c}\text { Frecuencia } \\
\text { (porcentaje) }\end{array}$ & $\begin{array}{c}\text { Peso/edad } \\
\%<-2 Z\end{array}$ \\
Marasmo & $10(2.2)$ & $90 \%$ \\
\hline Pérdida muscular en extremidades & $40(8.8)$ & $75 \%$ \\
\hline Alteraciones en cabello & $68(15.1)$ & $67 \%$ \\
\hline Edema en extremidades inferiores & $1(0.2)$ & - \\
\hline Bocio aparente & 0 & - \\
\hline Xerosis en conjuntiva (X 1A) & $11(2.4)$ & $82 \%$ \\
\hline
\end{tabular}

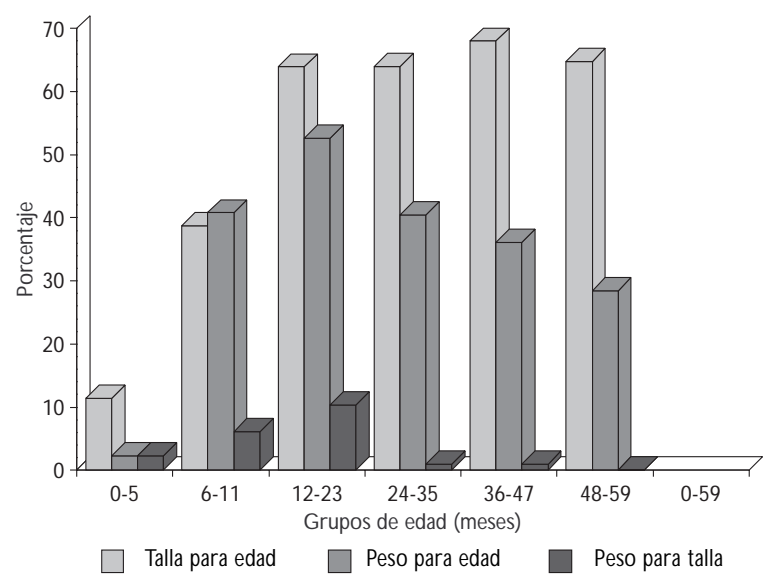

Figura 1. Porcentajes de tarahumaras menores de CINCO AÑOS A <-2Z PARA LOS INDICADORES ANTROPOMÉtricos, POR GRUPOS DE EDAD. GUACHOCHI, ChIHUAHUA, MÉXICO, 1997

salud pública de méxico / vol.42, no.1, enero-febrero de 2000 


\section{Discusión}

La imposibilidad de llevar a cabo un muestreo probabilístico dada la falta de un marco muestral así como los problemas logísticos para lograr un muestreo representativo son algunas razones por las que, no obstante el conocimiento de problemas nutricios en la zona, no se había realizado ningún estudio con base poblacional para conocer la prevalencia de desnutrición infantil tarahumara. Sin embargo, esta información es esencial para diseñar e instrumentar adecuadamente programas de intervención, y para evaluar el impacto de las acciones emprendidas. Es por esto que, a pesar de las limitaciones en la selección de la muestra, esta encuesta presenta una contribución importante para planear acciones de ayuda nutricional dirigidas a los niños tarahumaras.

Es conveniente hacer una consideración sobre el patrón de referencia sugerido por la OMS. Varios autores han cuestionado el uso de esta referencia en poblaciones de países en vías de desarrollo, ya que se basa en el patrón de crecimiento de los niños estadunidenses. ${ }^{25-27}$ Sin embargo, esta referencia no sólo es la única existente para hacer comparaciones internacionales, sino que, además, se ha mostrado que las principales variaciones en los patrones de crecimiento se deben a factores ambientales y socioeconómicos, más que a diferencias étnicas o raciales. ${ }^{28,29}$ Se ha notificado una variabilidad mundial de $1 \mathrm{~cm}$ en la talla de los niños de cinco años en los que no ha habido influencias ambientales adversas en el crecimiento. ${ }^{30}$

La moderada prevalencia de emaciación (peso/ talla <-2Z) encontrada ( $3.5 \%)$, responde probablemente a varias causas. Por un lado, se ha mostrado que los niños latinoamericanos tienden a presentar un mejor estado en este indicador comparado con los niños asiáticos o africanos. ${ }^{31}$ Se ha dicho que interpretar las bajas prevalencias de emaciación como indicativas de baja intensidad de desnutrición en estas poblaciones puede conducir a conclusiones equivocadas, por lo que incluso se ha sugerido revaluar los puntos de corte de acuerdo con la población..$^{25,26} \mathrm{Si}$ se compara esta prevalencia de 3.5\% dentro del contexto latinoamericano, ciertamente aparecerá como alta (debe notarse que esta cifra incluye a los niños de $0-5$ años). ${ }^{31}$ Por otra parte, las altas prevalencias de emaciación se asocian con el deterioro agudo del estado nutricional como resultado de hambrunas, enfermedades infecciosas u otros factores ambientales, producto de la caída de la distribución del indicador peso/talla no sólo en aquellos niños cercanos a los puntos de corte sino de todos los niños afectados. ${ }^{32}$ Aparentemente las con- diciones de producción durante 1995-1996 mejoraron en comparación con los años previos de sequía (1992-1994), en los que la productividad de maíz por hectárea fue calculada en $100-200$ kg* en las tierras de los tarahumaras lo que la hace insuficiente para la subsistencia. Fue precisamente durante 1994 cuando se desencadenaron los informes sobre el incremento en la mortalidad infantil tarahumara asociada con desnutrición en la región.

La elevada prevalencia de déficit de talla (talla/ edad $<-2 Z$ ) encontrada ( $57.1 \%$ ) es un predictor de riesgo que refleja el nivel general de desarrollo de la población. La baja talla adquirida hasta los cinco años limita la talla alcanzada en la edad adulta, lo que conlleva a una reducción en la capacidad laboral ${ }^{33,34}$ y a mayores riesgos obstétricos. ${ }^{35,36}$ En el análisis estratificado de la Encuesta Nacional de Nutrición (ENN), de 1988, se encontraron prevalencias similares de déficit de talla (cercanas a 60\%) sólo entre los niños indígenas hijos de madres con muy pobre educación formal, y cuyas viviendas se encontraban en el tercil más bajo de la clasificación socioeconómica. ${ }^{37}$

En cuanto a la diferencia encontrada por sexo, llamó la atención que los niños presentaran mayor afectación que las niñas. Las razones pudieran ser culturales. Aparentemente se espera que los niños sean más resistentes físicamente que las niñas. Esto se refleja en tradiciones tales como el juego tradicional de pelota entre los hombres, que exige una resistencia física enorme, en contraste con el juego del aro en las mujeres que demanda notablemente menor esfuerzo. Es posible que las madres presten menor atención a la alimentación de los niños por suponerlos más resistentes y que den preferencia a la alimentación de las niñas por considerarlas más vulnerables.

Las menores deficiencias nutricias, reflejadas en puntajes Z más altos, particularmente, en peso para la edad y talla para la edad, en relación con el tamaño de la localidad de residencia se encontraron en las rancherías grandes. Son estas rancherías las que tienen mayor disponibilidad de alimentos, de servicios de salud y educación, y de transporte, lo que podría explicar el menor déficit nutricio observado en estos lugares. Sin embargo, el hecho de que la magnitud de las diferencias no fuera muy grande, pudiera sugerir que el niño tarahumara es víctima de una marginación socioeconómica más que geográfica.

\footnotetext{
* Monárrez-Espino J. Estudio preliminar de desnutrición infantil tarahumara. Reporte SSA. Chihuahua: SSA, 1994. Documento mimeografiado.
} 
La proporción de niños con signos de desnutrición y puntuaciones a $<-2 Z$ para el indicador peso/ edad sugiere una correlación aceptable entre el diagnóstico clínico y el antropométrico. La ausencia de bocio aparente sugiere que la deficiencia de iodo no es un problema importante en este grupo de edad. Por otra parte, aun cuando la xerosis conjuntival no es considerada como un criterio aceptable para determinar si la deficiencia de vitamina A representa un problema de salud pública, ${ }^{38}$ la prevalencia de $2.4 \%$ encontrada debe tomarse en cuenta en estudios subsecuentes.

La prevalencia de desnutrición en relación con la edad señala claramente los grupos de mayor riesgo. La desnutrición, reflejada en los tres indicadores antropométricos, comienza a ser evidente desde edad temprana. En el comportamiento del peso para talla, que refleja un déficit presente, se encontró un claro incremento a partir de los seis meses, alcanzando su máximo en los niños de 12-23 meses que presentaron una prevalencia de emaciación de $10.2 \%$ (superando $8 \%$ que establecen las agencias internacionales como "emergencia nutricional"), para luego declinar, mostrando secuelas de desnutrición pasada (bajo peso para edad, no así de peso para talla) en el tercero, cuarto y quinto año de vida. El comportamiento de la talla para la edad muestra el déficit acumulado de desnutrición crónica: el porcentaje de niños con deficiencia $(<-2 Z)$ se elevó de $11.4 \%$ en el primer semestre de la vida a $38.8 \%$ en el segundo semestre, para alcanzar $63.9 \%$ en el segundo año de vida y mantenerse prácticamente igual hasta el quinto año.

Si se utiliza la clasificación de Gómez, que considera tres grados de severidad en función del porcentaje de déficit en comparación con el valor de la mediana de la población de referencia, ${ }^{39}$ para comparar los resultados de esta encuesta con los encontrados por la ENN, de 1988, en el ámbito nacional, ${ }^{40}$ se observa notablemente mayor afectación en los niños rarámuri. Para la encuesta tarahumara y la ENN, respectivamente: desnutrición leve $53.1 \%$ y $26.7 \%$; desnutrición moderada, $23.8 \%$ y $8.1 \%$; desnutrición grave, $1.3 \%$ y $0.7 \%$.

Aunque el diseño muestral empleado no garantiza la validez externa de los hallazgos, por lo que la generalización a otros municipios predominantemente tarahumaras en el estado es cuestionable, los resultados de este estudio pueden orientar las acciones para dirigir la ayuda hacia la población infantil tarahumara. Destaca la necesidad de concentrar la ayuda en los niños menores de dos años, no solamente en aquellos que cumplan con los criterios antropométricos de des- nutrición sino también en los que presenten signos clínicos de ésta.

De ofrecerse una canasta alimentaria directa como la que ha implementado el gobierno, debe contener alimentos para los niños pequeños, de preferencia con alimentos localmente disponibles y con aceptabilidad cultural, como ha sido sugerido por los autores. ${ }^{41}$ Además, es prioritario dirigir los programas de ayuda a las rancherías pequeñas, frecuentemente aisladas, con muy pobre acceso a servicios educativos y de salud.

Un hallazgo relevante desde el punto de vista antropológico, aunque de poca relevancia práctica, fue el de encontrar mayor deficiencia nutricia entre los niños que entre las niñas. Estudios en otros países en vías de desarrollo, particularmente en Asia, han informado un sesgo de género en cuanto a la preferencia por ofrecer una mejor alimentación a los hombres y una "discriminación" hacia las mujeres. En este estudio, la situación resultó a la inversa. ${ }^{42,43}$ Es deseable contar con información de tipo etnográfico que permita ahondar en las razones que están detrás de este hallazgo.

Cabe destacar que las dificultades logísticas enfrentadas en este estudio hacen poco factible la posibilidad de repetir una encuesta como la presente con cierta periodicidad que permita un monitoreo de la condición nutricia entre los indígenas tarahumaras. Sin embargo, es imprescindible contar con medidas que permitan evaluar el impacto de los programas ejercidos. Una alternativa viable es recurrir a la metodología de sitios centinela, que ha sido empleada con éxito para evaluar el impacto de intervenciones específicas de salud y nutrición en poblaciones de difícil acceso. ${ }^{44}$

Debe reconocerse que las influencias climáticas, las limitaciones de las superficies de labor y la accesibilidad a fertilizantes y plaguicidas son factores fundamentales que alteran el ciclo agrícola rarámuri y que determinan en buena medida las condiciones de nutrición y salud de la población. El equilibrio entre estos factores en la zona tarahumara es apenas sostenible y pequeños cambios en sus componentes pueden alterar la productividad de las cosechas y como consecuencia el estado nutricional de la población. Sin embargo, también debe reconocerse que los tarahumaras nunca han sido objeto de alguna intervención que haya incluido inversiones que favorezcan la creación de empleos y aumenten el nivel de ingresos de la población. Tampoco se ha tratado de desarrollar el sector alimentario y la escasa transferencia de alimentos y subsidios ha sido por demás insuficiente e inefectiva. Por esto, cualquier intervención puntual para comba- 
tir la desnutrición infantil, que no vaya acompañada de medidas generales de desarrollo, será incapaz de garantizar que ésta no sea recurrente.

\section{Reconocimientos}

Los autores agradecen la colaboración del Instituto Nacional de Estadística, Geografía e Informática-Chihuahua, de la presidencia municipal de Guachochi y de Impresión Gráfica. Un agradecimiento especial para Manfred Braig por su valiosa participación en la recolección de la información.

\section{Referencias}

1. Instituto $\mathrm{N}$ acional de Estadística, Geografía e Informática. Resultados definitivos del Conteo General de Población y Vivienda, Chihuahua 1995. México, D.F.: IN EGI, 1997.

2. Fernández P. Elementos para una caracterización socioeconómica de la población indígena. México, D.F.: SSA-CEPS, 1992.

3. G arcía E. Modificaciones al sistema de clasificación climática de Köppen y Geiger adaptada a las condiciones de la República Mexicana. México, D.F.: Universidad N acional Autónoma de México, 1973.

4. Kennedy J. Tarahumara of the Sierra Madre; beer, ecology and social organization. Arlington Heights: AHM Publishing Corporation, 1978.

5. Peña M. C hihuahua económico. C hihuahua, México: Gobierno del estado, 1950.

6. Pennington C. The Tarahumar of Mexico. Salt Lake City: University of Utah Press, 1963.

7. Monárrez-Espino J. Caracterización de la desnutrición infantil tarahumara y diseño de una canasta de ayuda alimenticia con aceptabilidad cultural. Cuernavaca, Morelos, México: Escuela de Salud Pública de México. Instituto N acional de Salud Pública, 1998.

8. Fernández P. La mortalidad infantil indígena en 1990: una estimación a través de los municipios predominantemente indígenas. México, D.F.: SSA-CEPS, 1992

9. Gómez J, Partida V. N iveles de la mortalidad infantil y fecundidad en México, por entidad federativa, 1990. México, D.F.: SSA-CEPS, 1992.

10. Tompkins $A, W$ atson F. Malnutrition and infection: A review. Londres: Clinical N utrition Unit, Centre for Human Nutrition, London School of Hygiene and Tropical Medicine (ACC/SCN State-of-the-art Series, N utrition Policy Discussion Paper N 0.5), 1990.

11. Pelletier DL, Frongillo EA, Habicht JP. Epidemiologic evidence for a potentiating effect of malnutrition on child mortality. Am J Public Health 1993:83(8):1130-1133.

12. Pelletier DL.The potentiating effects of malnutrition on child mortality: Epidemiologic evidence and policy implications. N utr Rev 1994; 52(12):409-415.

13. Brown KH, Black RE, Becker S. Seasonal changes in nutritional status and the prevalence of malnutrition in a longitudinal study of young children in rural Bangladesh. Am J C lin N utr 1982;36(2):303-312.

14. Babu SC, Thirumaran S, Mohanam TC. Agricultural productivity, seasonality and gender bias in rural nutrition: Empirical evidence from South India. Soc Sci Med 1993;37(11):1313-1319.

15. Branca F, Pastore G, D emissie T, Ferro-Luzzi A.The nutritional impact of seasonality in children and adults of rural Ethiopia. Eur J Clin Nutr 1993;47(2):840-850.
16. Lemeshow S, Hosmer D, Klar J, Lwanga S.A dequacy of sample size in health studies. West Sussex: John W iley \& Sons for W HO 1990.

17. United Nations. How to weigh and measure children:Assessing the nutritional status of young children in household surveys. N ueva York: Department of Technical cooperation for development and Statistical 0 ffice, 1986.

18. Habicht JP. Estandarización de métodos cuantitativos so bre el terreno. Bol 0 ficina Sanit Panam 1974;76(5):375-384.

19. N ational Center for Health Statistics. Growth Charts. Rockville, MD: US D epartment of Health, Education and W elfare, Public Health Service, Health Resources Administration, 1976.

20. $O$ shaung A, Pedersen J, Diarra M, Bendech M, Hatloy A. Problems and pitfalls in the use of estimated age in anthropometric measurements of children from 6 to 60 months of age: A case from Mali. J N utr 1994;124(5): 636-644.

21. Van-den-Broeck J, Meulemans W, Eeckels R. N utritional assessment: The problem of clinical-anthropometrical mismatch. Eur J C lin N utr 1994; 48(1):60-65

22. Jelliffe D. Evaluación del estado de nutrición de la comunidad. Ginebra: O rganización Mundial de la Salud, 1968.

23. Golden M. Severe malnutrition. 0 xford textbook of medicine. 0 xford: United Press, 1996:1278-1296.

24.W orld Health 0 rganization. Expert committee on physical status:The use and interpretation of anthropometry. Ginebra:W HO, 1995.

25.Victora C. The association between wasting and stunting:An international perspective. J N utr 1992;122(5):1105-1110.

26.Y ip R, Scanlon K, Trow bridge F.Trends and patterns in height and weight status of low-income US children. Crit Rev Food Sci N utr 1993;33(4-5): 409-421.

27. Martorell R, Mendoza FS, Castillo RO, Pawson IG, Budge CC. Short and plump physique of Mexican-American children. Am J Phys Anthrop 1987:73(4):475-487.

28. Habicht JP, Martorell R, Yarbrough C, Malina RE, Klein RE. Height and weight standards for preschool children. How relevant are ethnic differences in growth potential? Lancet 1974;1(7858):611-614.

29. Graitcer PL, Gentry EM. Measuring children: 0 ne reference for all. Lancet 1981;2(8241)1:297-299.

30. Martorell R. C hild growth retardation:A discussion of its causes and of its relationship to health. En: Blaxter $K, W$ aterlow J, ed. $N$ utritional adaptation in man. Londres: John Libbey, 1985:13-30.

31.0 nis M, Monteiro C,A kre J, G lugston G.The worldwide magnitude of protein-energy malnutrition: An overview from the W HO Global Database on Child Growth. Bull W orld Health O rgan 1993;71(6):703-712.

32. Herwalt BL, Bassett DC,Yip R,Alonso CR,Toole MJ.C Crisis in Southern Sudan:W here is the world? Lancet 1993;342(8863):119-120.

33. Martorell R. Long-term consequences of growth retardation during early childhood. En: Hernández M, Argente J, eds. Human growth: Basic and clinical aspects. Amsterdam: Elsevier, 1992:143-149.

34. Spurr GB, Barac-N ieto M, Maksud MG. Productivity and maximal oxigen consumption in sugar cane cutters. Am J C lin N utr 1977;30(3): 316-321.

35. Camilleri AP.The obstetric significance of short stature. Eur J 0 bstet Gynecol Reprod Biol 1981;12(6):347-356.

36. Harrison KA. Predicting trends in operative delivery for cephalopelvic disproportion in Africa. Lancet 1990;335:861-862.

37. Rivera-D omarco J, González-C ossío T, Flores M, Hernández-Avila M, Lezana MA, Sepúlveda-Amor J. D éficit de talla y emaciación en menores de cinco años en distintas regiones y estratos de México. Salud Publica Mex 1995;37(2):95-107.

38. Sommer A.Vitamin A deficiency and its consequences:A field guide to detection and control. Ginebra:W orld Health 0 rganization, 1995.

39. Gómez F, Ramos-Galván R, Cravioto J, Frenk S, Chávez R, Vázquez J. Mortality in second and third degree malnutrition. JTrop Pediatr 1956:2:77. 
40. Sepúlveda-A mor J, Lezana MA,Tapia-Conyer R,Valdespino J, Madrigal $H$, Kumate J. Estado nutricional de preescolares y mujeres en México: Resultados de una encuesta probabilística nacional. Gac Med Mex 1990; 126(3):207-225.

41. Monárrez-Espino J, Martínez H.An ethnographic approach to designing a food aid basket for children under three years of age in an indigenous area of N orthern Mexico. En: Köhler B, Feichtinger E, Dowler $E$, W inkler $G$, ed. Poverty and food in welfare societies. Berlin:W issenschaftszentrum Berlin für Sozialforschung, 1999:208-217.
42. Basu AM. How pervasive are sex differentials in childhood nutritional levels in South Asia? Soc Biol 1993;40(1-2):25-37.

43. Henry FJ, Briend A, Fauveau V, H uttly SA, Yunus M, C hakraborty J. Gender and age differentials in risk factors for childhood malnutrition in Bangladesh. Ann Epidemiol 1993;3(4):382-386.

44. Andersson N, Martínez E, Villegas A, Rodríguez I.Vigilancia epidemiológica y planificación descentralizada: el uso de sitios centinela en Guerrero. Salud Publica Mex 1989;31(4):493-502. 